\title{
DOCUMENTATION
}

\section{Le programme des grands travaux d'aménagement hydroélectrique à exécuter en Belgique pour l'utilisation des ressources nationales}

Maintenant qu'après huit années d'un effort admirable, elle est parvenue à relever ses ruines et à réparer les instruments de sa production, maintenant qu'elle a procédé - non sans s'être imposé de lourds sacrifices - à la stabilisation de sa monnaie, la Belgique a la ferme préoccupation d'adapter toutes les ressources de son territoire aux conditions nouvelles du progrès, pour reprendre définitivement la place qu'elle occupait avant la guerre au premier rang des nations industrielles. Il lui reste à perfectionner et à développer son outillagé économique en recherchant les facteurs qui, délaissés jusqu’ici, y peuvent contribuer le plus efficacement.

C'est dans ce but, et en vue de soutenir et de compléter le redressement monétaire par le redressement économique, que le gouvernement belge instituait, le 2 mars 1927, une Commission nationale des grands Travaux. Composée de 33 membres : ingénieurs, fonctionnaires du génie civil, industriels, hommes d'affaires éminents, elle avait pour mission l'examen, au triple point de vue technique, économique et financier, des travaux à exécuter pour améliorer l'accès vers la mer des centres industriels du pays, pour mettre le bassin de la Meuse à l'abri des inondations et pour résoudre les problèmes qui se rattachent à ces deux questions, notamment la production et la distribution d'énergie hydroélectricpue.

La commission s'est mise aussitôt à l'œuvre; ses membres, répartis en quatre sous-commissions, ont témoigné de la plus grande activité et du réel souci d'aboutir à des solutions à la fois hardies et réalisables; une cinquantaine de séances ont été tenues, plusicurs voyages effectués, en conclusion de quoi un rapport technique a été présenté par chacune des sous-commissions dont lensemble constitue le rapport général de la commission. Le toul forme un fascicule de près de cent pages, grand format : véritable programme détaillé de ce que doit être l'effort qui s'impose afin de tirer tout le parti possible des ressources nationales.

Voici, cl'après le compte rendu qu'en a donné M. E. Vielfaure dans "La Journée industrielle "les grandes lignes de ces travaux :

I. Aménagements muvvaux. - Le problème qu'avait à résoudre la commission comportait done deux parties : l'exploitation systématique des ressources hydrauliques et l'organisation rationnelle de la production d'électricité, - toutes deux connexes, puisque les rivières ardemaises et la Meuse peuvent donner de l'énergie ćlectrique et que l'organisation rationnelle de l'électricité est la condition nécessaire pour que l'on puisse tirer pleinement profit de cette énergie hydraulique.

L'exploitation systématique des ressources hydrauliques comporte, aux yeux de la commission, la création de barrages-réservoirs en Ardenne et l'aménagement de la Sambre et de la Meuse, qui doivent permettre à la fois la lutte contre les inondations, la récupération électrique et l'amélioration de la navigation.

" Il faut construire des barrages-réservoirs en Ardenne, explique la commission, car c'est pratiquement le seul moyen de disposer de l'eau nécessaire pour alimenter les canaux de la Meuse vers Anvers et c'est, au surplus, un moyen de produire de l'énergie électrique dans des conditions intéressantes. Actuellement, en effet, on ne dispose pas pendant la période des basses eaux de la quantité d'eau nécessaire pour ces voies navigables. Ce n'est qu'en emmagasinant de grandes réserves derrière des murs de barrage, pendant la saison pluvieuse, pour les restituer pendant la période sèche, que l'on pourra prendre à la Meuse la quantité d'eau nécessaire pour maintenir le niveau dans les canaux vers Anvers. Quant à la quantité d'énergie électrique que l'on pourra produire, elle est de l'ordre de 250 millions de kilowatts-heures pour l'Ourthe et l'Amblève et de un deni-milliard de kilowatts-heures pour la Semois et ses affluents \%.

Notons que la production totale actuelle de la Belgique est estimée à 3 milliards 160 millions de kilowatts-heures. En outre, les barrages régulateurs projetés mettront fin aux débordements des rivières ardennaises et contribueront ainsi à régulariser le débit de la Meuse, dont les crues, certaines années, sont de véritables désastres pour la région liégeoise.

Cependant, ces réservoirs ne sauraient suffire à mettre fin aux inondations. Aussi, le lit de la Sambre et celui de la Meuse devrontils être élargis en certaines sections et approfondis, ce qui favorisera du même coup le développement de la navigation et la récupération de l'énergie hydraulique; on pourra produire dans quatre ou cinq usines, au fil de l'eau, 130 à 185 millions de kilowatts-heures à des prix de revient avantageux.

En ce qui concerne la navigation sur la Sambre et sur la Meuse, la première, actuellement accessible à des bateaux de $430 t$, pourra recevoir des bateaux de $600 \mathrm{t}$ après l'exécution du programme dont la commission recommande la prompte exécution. Le bassin de Charleroi et la région industrielle de la basse Sambre doivent, en effet, être reliés à Liége et à tout le bassin de la Meuse par une large voie de communication. La Meuse peut devenir, à peu de frais, une magnifique voie navigable de Givet à Visé. De Givet à Ben-Ahin, les écluses de $100 \mathrm{~m} \times 12 \mathrm{~m}$, construites pour 4 bateaux de $300 \mathrm{t}$, peuvent laisser passer des bateaux jaugeant $1.350 \mathrm{t}$. Quand les travaux projetés seront achevés, la navigation pour bateaux de gros tonnage sera possible sur la Meuse dans toute la partie belge. Par la Sambre, la Meuse est en communication avec le bassin industriel de Charleroi ; par son cours supérieur, elle touche au bassin ferrifère de l'Est de la France et de la Lorraine, à la région de Nancy, au gisement des salines, au district de la Sarre et à une partie de l'Alsace. Si la navigation était améliorée et prolongée vers ces régions, par l'exécution des projets français, la Meuse deviendrait le trait d'union entre l'ensemble de tout ce pays industriel et la mer.

Le débouché vers la mer de cette voie navigable, appelée à prendre une si grande importance dans l'avenir, voilà le grave problème qui a retenu toute l'attention de la commission et qui est celui des voies navigables entre la Meuse et Anvers.

Il existe déjà, comme chacun sait, un canal Liège-Anvers, à travers la Hollande, par Mëastricht. La commission nationale des grands travaux estime que cette terminaison de la navigation mosane vers la mer ne répond plus aux nécessités actuelles. Le canal de la Meuse vers Anvers, accessible aux bateaux de $510 t$, est encombré à certains endroits, notamment à son entrée à Liége, du côté d'Anvers et surtout dans l'enclave de Maëstricht, où sont accumulées toutes les difficultés qui peuvent entraver la navigation et qui, peut-être, ne sont pas entièrement imputables la commission ne le précise évidemment pas - aux conditions géographiques et techniques.

Pour ces raisons, et aussi pour doter d'une voie d'eau le nouveau bassin industriel de la Campine, la Commission nationale des grands Travaux recommande la construction d'un canal direct, entièrement en territoire belge, sans bief de partage, pour bateaux de $1.350 \mathrm{t}$. La Meuse, ainsi jetée en quelque sorte dans l'Escaut, c'est la liaison directe et rapide entre le bassin industriel de Liége et la mer, par une distance de $125 \mathrm{~km}$. seulement. Rapprochement considérable des deux fleuves, si l'on songe que le trajet actuel entre Liége et Anvers réclame une dizaine de jours, alors qu'il n'en faudra pas mème deux par celui qui est prévu.

La durée d'exécution du nouveau canal exigerait huit à dix années, tandis que le coût des travaux est évalué entre 600 et 700 millions de francs belges (360 à 420 millions de francs français).

Quant au trafic dont il est susceptible, on l'estime de 8 à 10 millions de tonnes, au lieu de 2.500 .000 sur le canal actuel. C'est qu'en effet, et bien avant mème que cette voie soit achevée, les nouveaux charbonnages campinois produiront au moins 6 millions de tonnes de houille : charbon à coke et charbon de soute, qui s'en iront, l'un vers le sud alimenter les hauts fourneaux du bassin liégeois, de la Lorraine et de l'Est français, l'autre vers le nord, pour satisfaire à Anvers aux besoins des navires. La commission prévoit, en outre, qu'en apportant en Campine l'eau nécessaire à son industrie, le futur canal verra, sur ses deux rives, depuis Anvers jusqu'au sud du bassin houiller, de nombreuses usines venir s'établir attirées à la fois par la proximité d'Anvers, le voisinage des charbonnages et l'abondance de l'eau. 
Enfin, on a vu plus haut que, par la Meuse convenablement aménagée, une autre fonction lui était assignée, tout aussi imperieuse : celle d'amener dans la sphère d'attraction du port d'Anvers de gros éléments du trafic mosan qui prenment leur source dans l'Est français, en Lorraine, en Alsace et dans la Sarre. Qui n'apercoit, en effet, l'avantage tout d'un coup offert par cette voie facile au ravitaillement et aux exportations de ces régions si industrielles ?

Faire d'Anvers le point d'aboutissement de la navigation mosane, telle a été la préoccupation de la Commission nationale des grands Travaux. Ses conclusions seront fàcheusement ressenties en Hollande, qui aspirait au mème but en faveur de Rotterdam. Elle entendait mème $y$ atteindre au plus tôt, puisque la canalisation de la Meuse, jusqu'à l'endroit où elle touche à la Belgique, est déjà en voie d'achèvement, tandis que sont commencés les travaux du canal Juliana, qui, raccordant le Rhin à la Meuse, amènera à Rotterdam le trafic de celle-ci. Soixante-dix millions de florins ont, dit-on, été déjà investis dans cet important ouvrage auquel le canal direct Liége-Anvers enlèvera désormais une partie de son utilité. C'est là une des conséquences du rejet par le Parlement néerlandais du traité hollando-belge.

Quant aux répercussions que la voie Givet-Anvers est appelée à avoir sur les régions de tout l'Est français, dont, à travers la Belgique, elle drainera une bonne partie du ravitaillement et des exportations au profit d'Anvers, elles paraissent devoir ètre assez profondes, à la fois heureuses et fâcheuses pour notre pays. Heureuses, si l'on ne considère que l'intérêt propre des départements qu'elle desservira; fàcheuses si l'on pense au trafic énorme dont elle privera désorniais nos chemins de fer et nos ports. Et l'on peut prévoir que Dunkerque s'en trouvera tont aussi affectée que Rotterdam.

II. Aménagement pour la production de l'Électricité. Le deuxième problème qu'avait à résoudre la commission était, nous l'avons noté, l'organisation rationnelle de la production de l'électricité. "Dans un pays comme le nôtre, explique le rapprot présenté à ce sujet, dont la population est dense et dont la production agricole et industrielle est importante, l'énergie électrique doit être distribuée partout et aux meilleures conditions possibles. L'électricité à bas prix améliorera la situation de l'industrie, permettra aux producteurs belges de lutter plus facilement sur les marchés internationaux et rendra ainsi la Belgique plus prospère.

Le programme général d'exécution, d'organisation et d'exploitation des installations comporte notamment, ainsi qu'il résulte de ce qui est dit plus haut, la construction d'usines hydroélectriques dépendant des barrages-réservoirs, et le fonctionnement de ces usines, de ce concert avec des usines au fil de l'eau établies sur la Meuse, qui devraient être connectées aux premières et à des usines thermiques.

Le plan de la commission repose sur quatre points principaux : 10 la concession des forces hydrauliques d'un même cours d'eau ou d'un ensemble de cours d'eau, soit à un organisme unique, soit à un groupement de sociétés industrielles; $2^{\circ}$ l'établissement des barrages et des usines de façon à réaliser le plus possible les buts principaux d'intérêt public (atténuation. des inondations, alimentation d'un canal vers Anvers, etc), tout en assurant la plus grande valeur possible à l'énergie électrique distribuable; $3^{\circ}$ la liaison des usines hydrauliques, à créer, avec des usines thermiques (ou des groupements d'usines thermiques, cokeries et hauts foumeaux), ces usines therniques étant soit des usines existantes, soit des usines génératrices au charbon, à construire pourvues de groupes électrogènes de grande puissance; $4^{\circ}$ le groupement des exploitants des diverses usines interconnectées (hydroélectriques, thermiques actuelles et futures), ou accord pour l'exploitation en commun de ces usines, des lignes d'interconnexion, de manière à réduire au strict minimum la consommation de combustible et le prix de revient de l'énergie distribuée.

L'organisation rationnelle de la production clectrique permettra aux métallurgistes belges de tirer parti en tout temps de leurs excès de gaz et de produire une quantité supplémentaire d'électricité, qui serait, en un an, l'équivalent de 1.200 .000 t. de houille. En 1935, selon les prévisions généralement admises, la consommation d'énergie électrique sera vraisemblablement en Belgique le double de ce qu'elle est actuellement. Grâce a la rationalisation, il sera possible de produire cette quantité double sans brûler une tonne de chargon de plus. La consommation de houille, quì serait pour la marche indépendante de $6.850 .000 \mathrm{t}$., ne sera que de 3.060 .000 t. De plus, grâce aux économies réalisées dans l'utilisation des sources d'énergie, dans l'utilisation du courant et dans les immobilisations nouvelles, le prix de revient du kilowattheure moyen aura été abaissé de 40 à 25 centimes.

III. Exécution et financement des travaux. - Tel est le résumé du programme élaboré par la Commission nationale des Grands Travaux et qui ne manque certes pas, de hardiesse. En le faisant sien et en le déposant sur le bureau du Parlement, le gouvermement a marqué son intention de le conduire promptement dans le domaine de la réalisation. "Ce plan, a dit à la Chambre M. Jaspar, premier ministre, assure la continuité de l'œuvre entreprise. Cette continuité comme la rapidité de l'exécution des travaux, sont une nécessité impérieuse. Tous sont importants, tous sont nécessaires; certains sont d'une urgence extrème. Les uns et les autres participent au programme sur lequel le gouvernement s'est constitué; sa réalisation justifie à la fois la composition de ce gouvernement et son existence, conditionnées toutes deux par la nécessité de rétablir la richesse et la puissance de notre pays."

Les services juridiques des ministères des Finances et des Travaux publics ont élaboré cinq projets de loi : projet sur le fonds national des grands travaux et les ressources qui l'alimenteront; sur les concessions de forces hydrauliques à accorder ; sur l'organisation de la navigation fluviale; sur la production de l'énergie électrique; sur la création du fonds national des routes.

Des estimations, soigneusement établies et contrôlées, permettent d'évaluer approximativement à 2 milliards de francs le total des dépenses à faire pour exécuter les travaux ci-dessus énumérés, y compris la réfection du réseau routier. Ce total s'échelonnerait sur une durée de douze à quinze années, temps jugé nécessaire pour l'achèvement du programme complet. Pendant les cinq premières années, les dépenses seront de l'ordre de 300 millions de francs; elles diminueront ensuite assez rapidement. Il sera fait face au total de 2 milliards ainsi répartis par les recettes extraordinaires de l'Etat. Afin d'assurer à l'exécution des travaux la continuité de leur achèvement, le gouvernement proposera la création d'un organisme autonome et de caractère public, en faisant appel aux compétences nécessaires, de manière à assurer la prépondérance des intérèts généraux du pays. L'Etat remettra, à cette fin, à cet organisme, soil les disponibilités budgétaires nécessaires, soit, en cas d'insuffisance, cles instruments de crédit.

\section{Un accumulateur merveilleux}

On a fait grand bruit, ces temps derniers, dans la presse quotidienne sur la soi-disant découverte d'un accumulateur électrique merveilleux capable de révolutionner les applications de ces appareils.

Nous avons reçu de nombreuses demandes de nos lecteurs relativement à cet accumulateur, et avant de répondre, nous avons tenu à nous documenter afin de pouvoir donner des renseignements en toute impartialité.

Nous ne pouvons mieux faire que de donner la traduction d'un article paru dans l'E.T.Z. (t. XLVIII, p. 950), journal extrêmement sérieux qui met la chose au point :

"Tout récemment, une série d'articles ont été publiés dans les journaux quotidiens nationaux et étrangers, sur l'invention d'un accumulateur électrique par le Père espagnol Almeida.

"Il s'agit d'un accumulateur dont l'électrode positive consiste en charbon ou graphite recouvert d'argent et dont j'ćlectrode négative est en zinc, tandis que l'électrolyte est un mélange de bromure de zinc et de chlorure de zinc. De semblables accumulateurs ont été plusieurs fois proposés et en particulier discutés d'une manière détaillée par M. L. Jumeau dans son livre "Les Accumulateurs électriques ", Paris 1907, pages 534 et suivantes.

"Dans ces articles, on a signalé qu'à l'Accumulatoren-Fabrik A. G. dans son usine de Hagen et plus tard à Berlin, les essais effectués avaient donné les résultats les plus satisfaisants.

"Nous nous sommes par conséquent adressés à l'AccumulatorenFabrik A. G. en la priant de nous renseigner. Cette firme nous a alors fait la communication suivante :

Il est de fait que l'Accumulatoren-Fabrik A. G., comme elle a coutume de le faire pour toutes les nouveautés qui lui sont offertes, a aussi essayé l'accumulateur Almeida, en présence et 
d'après les instructions de l'inventeur. Elle a dû pourtant, après 6 mois d'essais méticuleux, se décider à abandonner tous essais ultérieurs avec cette espèce d'accumulateur, parce qu'elle avait acquis la conviction qu'un résultat économique ne peut ètre obtenu avec une telle construction.

"D'après les déclarations des journaux, on doit obtenir à égalité de poids une énergie atteignant jusqu'à dix fois celle de tous les accumulateurs électriques employés aujourd'hui dans la pratique. Au lieu de cela, les essais mentionnés, très prolongés et rigoureux, ont prouvé indubitablement que l'énergie de l'accumulateur Almeida, par rapport à son poids, est notablement moindre que dans les accumulateurs au plomb et les accumulateurs alcalins couramment employés en pratique, pour des applications spéciales. Il en va de même aussi pour le rendement. Le Père AImeida n'a pas réussi non plus pendant le temps ues essais à exécuter une construction qui garantit une durée un peu longue. Tous les nombreux appareils réalisés d'après ses données ont prouvé qu'ils ne remplissaient pas leur but.

"Finalement, la raison qui fit décider l'abandon des essais est l'impossibilité d'éviter les vapeurs de brome et de chlore se dégageant en grande quantité pendant la charge de l'accumulateur, et très nuisibles pour la santé. Il n'existe d'ailleurs aucune probabilité que l'on pourrait réussir à éviter cet inconvénient dans l'application pratique de telles batteries.

"En abandonnant les essais, l'Accumulatoren-Fabrik A. G. s'en est remise au jugement du Père Almeida pour que celui-ci vienne la retrouver dès qu'il aurait obtenu quelque résultat intéressant au sujet de l'énergie et qu'il aurait supprimé les vapeurs nocives. Jusquu'à présent, des communications n'ont pas été faites par lui dans ce sens."

Comme on le voit, il $\mathrm{g}$ a loin de la coupe aux lèvres, il n'est certainement pas impossible que l'on réalise un jour l'accumulateur léger tant cherché, mais il faut encore patienter.

Les journaux à grand tirage qui avaient été jusqu'à dire que la Compagnie d'Orléans avait arrêté son électrification dès qu'elle avait appris la découverte du fameux accumulateur, font le plus grand tort aux inventions de ce genre. La Compagnie d'Orléans a bien suspendu ses travaux d'électrification, mais la raison est toute autre, e'est simplement à cause d'un manque de crédits, car si elle reprend ses lignes électriques ce sera toujours avec le système déjà adopté du courant continu à $1500 \mathrm{v}$.

Industrie Electrique, 10 décembre 1927.

A. S.

\section{L'importance et l'utilisation de l'énergie du Rhin; les usines hydroélectriques de Kembs et du grand canal d'Alsace}

La loi concédant à la Société des forces motrices du HautRhin les travaux d'aménagement du barrage sur le Rhin et de la chute de Kembs vient d'être votée en juillet 1927. C'est un événement d'une importance considirable puisqu'il correspond à la réalisation du premier échelon du vaste projet du grand canal d'Alsace pour la navigation et l'utilisation de la force hydraulique du Rhin.

L'auteur trace l'exposé de ce projet en commençant par l'étude du régime juridique très spécial auquel est soumis le Rhin, frontière de plusieurs états, et voie de navigation essentiellement internationale. C'est en 1792, à la Révolution Française, qu'il faut remonter pour en trouver la première base, dans la proclamation de la "libre navigation ", mais il n'y eut de réglementation établie que dans le traité de Paris (1814) et au Congrès de Vienne (1815). La "Commission rhénane "se réunit à Mayence en 1916 et continue depuis a siéger, rassemblant les membres des diverses nations qui touche le Rhin, à l'exception toutefois de la Suisse qui en fut exclue, ainsi que de la convention de Mannheim (1868) et dont on ne voit paraît-on de représentant dans les questions rhénanes qu'en 1919 à la commission centrale instituée par le traité de Versailles. Celui-ci (art. 358) institue au profit de la France : $1^{\circ}$ le droit de prélever sur le débit et d'effectuer les travaux nécessaires sur la rive allemande et $2^{\circ}$ le droit exclusif à l'énergie produite, sous réserve de payement à l'Allemagne, soit en argent, soit en énergie, la moitié de sa valeur. La commission centrale du Rhin eut son siège transféré à Strasbourg.

Des difficultés techniques considérables à surmonter, des tiraillements d'intérêts opposés entre les divers pay's intéressés allant jusqu'à l'obstruction systématique de la part de certains de ceux-ci voilà l'histoire de la correction de la navigabilité du Rhin jusqu'en 1918. Aujourd'hui, et depuis fort peu de temps, cette situation s'est éclaircie et notre pays a maintenant les mains libres, l'ère des pourparlers internationaux est terminée.

La Section Strasbourg-Bâle, qui, seule est en vue dans cette étude est celle qui présente le plus de difficultés : le Rhin y prend son équilibre du pays de plaine, avec de nombreux méandres en terrain instable et néanmoins la pente et la vitesse sont très fortes. De plus, des travaux de corrections faites en aval entre 1817 et 1876 ont fait apparaitre un redoutable travail d'éroption en profoncleur qui a provoqué l'affleurement du banc de roche transversal dénommé barre d'Istein, obstacle insurmontable à une correction efficace de la navigabilité par régularisation du cours du fleuve. Cette solution rendrait d'ailleurs impossible toute utilisation, pour la production de l'énergie, des 107 mètres de différence de niveau existant entre Bâle et Strasbourg.

L'autcur examine les deux solutions acceptables pour l'aménagement de cette section : canalisation du lit, en bief avec barrages et écluses ou établissement du canal latéral à grande section et fait ressortir les avantages de cette dernière, qui fut présentée en 1902 par M. Kochlin, a la Compagnie industrielle de Mulhouse et fit l'objet d'une demande en concession. Ce projet initial fut remanié et, dès 1910 , s'était constituée à Mulhouse la Société des
Forces Motrices du Haut-Rhin pour demander la concession de l'usine de Kembs, première partie du projet.

Ce projet, qui porte le nom de grand Canal d'Alsace, fut repris après le traité de Versailles, approuvé par le conseil supérieur des travaux publics et par la commission centrale du Rhin en mai 1922.

L'auteur décrit rapidement l'ensemble du projet, en faisantrapporter le lecteur aux cartes jointes à l'article. Le travail comporte 8 écluses et un seul barrage sur le Rhin (alors que la canalisation du lit même exigerait 14 écluses et autant de barrages, prolongés de chaque côté jusqu'au degrés des hautes eaux). A chaque écluse correspond une usine hydroélectrique. On trouve, l'amont en aval, les écluses aux usines de : Kembs, Ottmasheim, Fessenheim, Vogelgrün, Markalsheim, Sundhouse et Strasbourg, d'où la rentrée au Rhin s'effectue par le chenal d'accès au nouveau port.

Le débit prélevé sur le Rhin sera, au maximum, de $850 \mathrm{~m}^{5} / \mathrm{s}$. (pendant 7 mois); la longueur totale étant de $110,930 \mathrm{~km}$. et la vitesse n'étant nulle part supérieure à $1,20 \mathrm{~m}$./s. Les grands chalands du Rhin pourront y circuler avec leur charge de $1.200 \mathrm{t}$. admise en aval de Strasbourg. L'exécution de ce travail se fera par étapes, comportant chacune l'aménagement d'un bief, de l'usine correspondante, et d'un canal de fuite provisoire de raccordement au Rhin. Toutes les usines sont d'ailleurs prévues semblables.

L'auteur aborde ensuite la description de la première partie du projet, dont la réalisation va être entreprise ; le barrage du Rhin, origine de tout l'ouvrage, et l'usine de Kembs, extrémité du premier bief.

Le barrage, situé à $5,5 \mathrm{~km}$. en aval de la frontière Suisse aura 6 travées, les 3 centrales étant munies de vannes Stovey, pour répondre aux obligations spécifiées au traité de Versailles concernant l'écoulement des crues. Le seuil sera établi à la côte 232,5 , les fondations étant descendues dans la couche d'argile bleue compacte, à peu de profondeur au-dessous du gravier. Le niveau du Rhin sera surélevé de $8 \mathrm{~m}$. en basses eaux, ce qui portera l'origine de la courbe de remous à l'embouchure de la Boix. Cette surélévation se réduira à zéro en hautes eaux. Les travaux exécutés par le gouvernement français seront remboursés par les exploitants des usines au prorata de leurs concessions.

Les travaux de l'usine de Kembs incombent, par contre au concessionnaire, ainsi que ceux du canal d'amenée, de $5,6 \mathrm{~km}$. premier tronçon du grand canal d'Alsace; naissant à $500 \mathrm{~m}$. en amont du barrage, ce canal aura une largeur au plafond de $80 \mathrm{~m}$. et au plan d'eau de $150 \mathrm{~m}$. environ. Les couronnements des digues seront à la côte 245,50 , soit $1 \mathrm{~m}$. 50 au-dessus de la plus haute crue connue.

L'usine répondra au type usine-barrage. Utilisant une chute variant de $10 \mathrm{~m} .76$ à $15 \mathrm{~m} .68(13,32$ en eaux moyennes) elle comportera 8 groupes de 10 à $12.000 \mathrm{C}$. V. tournant à $93,8 \mathrm{t} . / \mathrm{m}$., composés d'une turbine Francis en chambre d'eau spirale avec aspirateur et d'un alternateur à axe vertical 
Le massif des chambres d'eau, fondé sur l'argile bleuc, présentera cette particularité d'offrir au-dessus de chaque cellule, entre la chambre d'eau ct le plancher de la salle des machines, un passage d'eau en déversoir obturé par une vanne automatique système Kiochlin-Logcher, permettant l'évacuation du débit en cas darrèt du groupe correspondant et le passage des corps flottants (glaces, branchages). Un déversoir est donc inutile dans cette disposition.

A l'usine sera accolée l'écluse de grande navigation (largeur $25 \mathrm{~m}$., longueur $185 \mathrm{~m}$. permettant le passage d'un train composé d'un remorqueur et 2 chalands). De plus, l'espace sera ménagé pour lemplacement éventuel d'une deuxième écluse de $25 \mathrm{~m}$. sur $100 \mathrm{~m}$. A l'amont de l'écluse un élargissement du canal ménagera un garage de $1.000 \mathrm{~m}$. de longueur, séparé par un guideau du canal d'arrivée de l'usine. A l'aval, un garage semblable de
$500 \mathrm{~m}$. existera. Le canal de fuite provisoire, très court, sera profondément encaissé.

L'auteur aborde ensuile l'étude ćconomique du projet el l'utilisation de l'énergie du Rhin dans la région de l'Est. En se basant sur une augmentation de 8 a $10 \%$ par an (soit de cloublement de la durée de présence en dix années) il est amené à conclure qu'en 1935 il faudrait se borner à la réalisation des 5 premières usines pour avoir une utilisation donnant un prix de revient acceptable au kw. h. D'ailleurs l'effort fusionné limitera à 3 le nombre d'usines qu'il sera possible de construire dans ce délai. Les 5 usines en question devront être réalisées dans un programme s'étendant sur 15 amnées, et en outre, on sera amené à prévoir une très grande extension des réserves thermiques ou des liaisons avec des bassins hydrauliques à régime différent, pour la régularisation du régime glaciaire du Rhin. R. G. E., 10 sept. 1927.

\section{L'usine génératrice d'East River de la New-York Edison $C_{y}$}

Cet article accompagné de plusieurs plans et coupes, est inspiré d'une monographie publiée par la General Electric Review.

La construction de cette puissante centrale a été motivée par le rapide accroissement de la charge maxima des réseaux de l'Edison $\mathrm{Cy}$ et de l'United light and Power $\mathrm{Cy}$ desservant la ville de New-York, charge qui de 400.000 wk. en 1920 atteint 732.000 en 1926.

L'usine en question est prévue pour une puissance totale de 1 million de $\mathrm{kw}$. ; la première branche $(120.000 \mathrm{kw}$.) a été mise en service en novembre 1926.

Le courant est actuellement fourni sous forme triphasée à 25 p. s. ; dans l'avenir, la fréquence de 60 p. s., déjà en service sur une partie des réseaux de New-York sera adoptée, et dès maintenant une puissante installation de convertisseur de fréquence est en service dans cette usine.

Le terrain disponible, en bordure de l'East-River qui présente en cet endroit une profondeur de $9 \mathrm{~m}$., permettant l'accostage des grands navires de mer, a une longueur de $488 \mathrm{~m}$. sur 244 de largeur. Il est à peu près au centre géographique du réseau à 25 p. s. de l'Edison.

Le bâtiment de la centrale est à 3 travées : la chaufferie, de $31 \mathrm{~m}$. de largeur, s'étendant sur $280 \mathrm{~m}$. de long, dans le pont définitif et actuellement sur $66 \mathrm{~m}$., la salle des machines qui a $17 \mathrm{~m} .80$ de la geur et une longueur actuelle de $75 \mathrm{~m}$. qui sera portée à $204 \mathrm{~m}$. 50 et la travée d'appareillage de $14 \mathrm{~m}$. de largeur sur $8 \mathrm{~m}$. (320 m. le projet achevé).

Un bâtiment annexe renferme l'installation de pulvérisation et de manutention du charbon.

Les fondations reposent sur des pieux en bois battus à une profondeur de $3 \mathrm{~m}$. 10, sur lesquels est établi un radier général en béton de $2 \mathrm{~m}$. 15 d'épaisseur.

Les chaudières seront au nombre de $44: 10$ sont actuellement installées desservies par 2 cheminées à virage induit. Elles sont du type Springfield, de $1.380 \mathrm{~m}^{3}$ (surchauffeur non compris) et fournissant $22.700 \mathrm{~kg}$. de vapeur à l'heure, et, en allure de pointe $113.500 \mathrm{~kg}$. La vapeur est produite à $26 \mathrm{k}$. 5 , à $370^{\circ}$.

Les foyers à charbon pulvérisé ont leurs parois constituées en écrans de tubes jointifs.

2 turbos de $60.000 \mathrm{kw}$. sont actuellement installés. Ils tournent à $1.500 \mathrm{t}$./s. et fournissent le courant à 11.400 volts, $25 \mathrm{p}$. s.

L'usine complétée comprendra 9 groupes, les 7 suivants étant prévus de $120.000 \mathrm{kw}$.

Le groupe convertisseur de frécuences $25 / 60$ p. s. actuellement installé comprend une machine synchrône à pôles saillants de $27.000 \mathrm{kw}$. à $25 \mathrm{p}$. s. accouplée à une génératrice asynchròne de $40.000 \mathrm{kw}$. à $60 \mathrm{p}$. s. I.e stator de la machine synchrône peut tourner sur lintervalle d'un pas polaire, au moyen d'un servo moteur à huile, pour la répartition de la charge avec le convertisseur de fréquence de $35.000 \mathrm{kw}$. installé à l'usine d'Hell-Gate. En bout d'arbre sont, d'un côté, l'excitatrice, de l'autre un moteur d'induction de $1,600 \mathrm{C} . \mathrm{V}$. pour le démarrage.

Les services auxiliaires principaux sont commandés par turbines à vapeur, les autres par moteurs électriques alimentés par 2 réseaux alternatifs $B$. T. et un réseau continu comportant 2 groupes turbos de $500 \mathrm{kw}$.

Le bâtiment de l'appareillage est établi sur le principe de la séparation des phases, un étage distinct étant occupé par chacune d'elles.

Le rez-de-chaussée est occupé par les transformateurs, notamment ceux de 2 fréquences du groupe convertisseur qui est couplé sur les 2 réseaux par l'intermédiaire de transformateur, les $3 \mathrm{e}, 4^{\mathrm{e}}$ et $5^{\mathrm{e}}$ étages sont accupés chacun par une des phases des circuits principaux à 11.100 volts.

Les appareils de manœuvre des interrupteurs occupent le $6^{\mathrm{e}}$ étage, à une extrémité de celui-ci, une salle ayant vue sur la salle des machines groupe les pupitres de commandes de tous les appareils $H$. T

Les départs de feeders sont groupés par 2 sur un interrupteur sélecteur. Tous les interrupteurs, des génératrices et des cléparts, sont établis pour une puissance de rupture de $1.500 .000 \mathrm{kw} \mathrm{A}$,

Les barres générales sont sectionnées en éléments correspondant chacun à 12 feeders et une génératrice. Des réactions limiavec chambre de compression.

tant le court-circuit instantané sont placées entre les sections et sur tous les départs et arrivées aboutissant aux barres.

Les barres sont logées dans des caniveaux en béton avec couvercles, et des précautions spéciales ont été prévues pour limiter la propagation des incendies.

Le point neutre du réseau est connecté à la terre par l'intermédiaire de transformateurs bobinés en zig-zag placés sur chaque section de jeux de barres.

Il est assez curieux de comparer le dessin de cette usine avec reux de nos grandes centrales francaises : la salle des machines surtout paraît réduite à un couloir étroit (malgré ses dimensions effectuées) où les groupes sont placés dans le sens de la longueur.

\section{Derniers progrès réalisés, en Suède, dans la construction des turbines hydrauliques}

Le rapporteur décrit les turbines construites à Trollhâttan, lesquelles sont munies d'un réglage intérieur combiné avec un réglage extérieur. Après avoir comparé les courbes de rendement des turbines de Queenston et de Porjus, il fait remarquer que la turbine à une seule roue à axe vertical, avec bâche en béton, est de plus en plus en faveur en Suède et que les types à rotation rapide (Kaplan, Lawaczek, Dahl) se répandent de plus en plus. Il termine par l'examen des dispositions particulieres aux turbines de l'usine de Norrforsen.

Rapport No 31 présenté à la section A de la deuxième Confírence mondiale de l'Energie (Bâle, 1926).

\section{L'utilisation de l'accumulateur de vapeur Ruths et la coopération de l'énergie hydraulique et de l'énergie thermique}

L'auteur expose rapidement les avantages des accumulateurs de vapeur utilisés en combinaison avec les groupes à vapeur ordinaires et même avec les usines hydroélectriques. Il décrit ensuite l'accumulateur Ruths et donne quelques détails sur les installations réalisées en Suède, aux usines électriques de Malmö et de Halmstad. On compte, d'autre part, en Scandinavie, une trentaine d'installations avec accumulateurs de vapeur réalisées dans des établissements industriels; l'auteur décrit deux de ces installations,

Rapport No 34, présenté à la section $\mathrm{C}$ de Johannes RuTHs. rence mondiale de l'Energie (Bâle, 1926). 